Slavica

bruxellensia

\section{Slavica bruxellensia}

Revue polyphonique de littérature, culture et histoire

slaves

5 | 2010

Après 1989

Krzysztof Wrocławski, Magdalena Bogusławska \& Ewa Wróblewska-Trochimiuk, Polska i Macedonia. Bibliografia. Komentarze. Studia.

\title{
Marek Pandera
}

\section{(2) OpenEdition}

Édition électronique

URL : http://journals.openedition.org/slavica/324

DOI : $10.4000 /$ slavica.324

ISSN : 2034-6395

Éditeur

Université libre de Bruxelles - ULB

Édition imprimée

Pagination : 90-91

ISSN : 2031-7654

Référence électronique

Marek Pandera, « Krzysztof Wrocławski, Magdalena Bogusławska \& Ewa Wróblewska-Trochimiuk, Polska i Macedonia. Bibliografia. Komentarze. Studia. », Slavica bruxellensia [En ligne], 5 | 2010, mis en ligne le 09 juillet 2012, consulté le 22 septembre 2020. URL : http://journals.openedition.org/slavica/ 324 ; DOI : https://doi.org/10.4000/slavica.324

Ce document a été généré automatiquement le 22 septembre 2020.

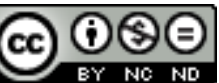

Les contenus de Slavica bruxellensia sont mis à disposition selon les termes de la Licence Creative Commons Attribution - Pas d'Utilisation Commerciale - Pas de Modification 3.0 France. 


\title{
Krzysztof Wrocławski, Magdalena Bogusławska \& Ewa Wróblewska- Trochimiuk, Polska i Macedonia. Bibliografia. Komentarze. Studia.
}

\author{
Marek Pandera
}

\section{RÉFÉRENCE}

Wrocławski Krz., Bogusławska M. \& Wróblewska-Trochimiuk E., Polska i Macedonia. Bibliografia. Komentarze. Studia, Varsovie, Instytut Slawistyki Zachodniej i Południowej Uniwersytetu Warszawskiego, Varsovie, 2009, 414 p.

1 Krzysztof Wrocławski, auteur de plus de cent publications scientifiques sur la langue macédonienne, s'est attelé, cette fois avec deux autres slavistes varsoviens, à la rédaction d'un recueil bibliographique, véritable synthèse de ce qui unit jusqu'à ce jour les Polonais et les Macédoniens sur le plan scientifique et culturel.

2 Ce travail se décline en trois parties principales. Dans la première partie, intitulée «Studia », les auteurs présentent l'univers, ô combien compliqué, des relations polonomacédoniennes. Ainsi, le lecteur peut non seulement se familiariser avec le profil historique des contacts entre la Pologne et la Macédoine, et ce dès l'époque du MoyenÂge, mais également avec l'évolution et la dynamique même des relations entre les deux pays. En outre, les auteurs ont décidé de présenter, de manière plus détaillée, le genius loci polono-macédonien en trois catégories d'intérêts: la linguistique, les contacts littéraires et les liens théâtraux. Une telle approche permet au lecteur non averti de découvrir clairement et rapidement la genèse relativement longue des relations scientifiques et culturelles entre ces pays, mais aussi de prendre la mesure de leur importance, de leur diversité et de leur complexité. 
3 La deuxième et la troisième partie (« Macedonica » et « Polonica ») traitent, tour à tour, de l'aspect bibliographique en signalant les ouvrages macédoniens publiés en Pologne et les ouvrages polonais parus en Macédoine. Du reste, les auteurs se concentrent délibérément sur les ouvrages slaves en les singularisant parmi les publications non slaves.

4 Il convient, ici, de rendre un hommage particulier tant à la richesse du matériel bibliographique rassemblé qu'à son agencement. Les données ont fait l'objet d'une collecte efficace et d'un minutieux ordonnancement, ce qui permet au lecteur de trouver aisément et rapidement des références bibliographiques intéressantes. Par ailleurs, les auteurs ont établi des listes thématiques consacrées aux travaux linguistiques, aux articles littéraires spécialisés ainsi qu'aux traductions littéraires. Le système de renvois utilisé par les auteurs est particulièrement utile dans la recherche de sources car il établit un lien entre les références bibliographiques et les commentaires, en indiquant les liens thématiques et les relations entre les différents travaux de recherche.

Dès lors, Polska i Macedonica. Bibliografia. Kommentarze. Studia constitue, d'une part, un précieux outil pour les spécialistes des sujets polono-macédoniens, et permet d'autre part au lecteur-amateur de s'initier, sans trop de difficultés, à la problématique, de l'appréhender, grâce aux publications réunies, dans le vaste contexte des relations bilatérales historiques, scientifiques et culturelles.

\section{INDEX}

Mots-clés : histoire de la Macédoine, histoire de la Pologne, littérature macédonienne, littérature polonaise

Index géographique : Macédoine, Pologne

\section{AUTEURS}

\section{MAREK PANDERA}

Lecteur de polonais à l'Université Libre de Bruxelles (Belgique), détaché de l'Université Jagellone (Cracovie, Pologne) 